\title{
Filtros de vena cava inferior en posición suprarrenal
}

\author{
Leopoldo Mariné $M$, Renato Mertens $M$, Albrecht Krämer \\ Sch, Francisco Valdés E, Michel Bergoeing R, Ivette \\ Arriagada J, Jeanette Vergara $\mathrm{G}$ a, Claudia Carvajal $\mathrm{N}^{\mathrm{a}}$. \\ Suprarenal inferior vena cava filters.
Retrospective review of 30 cases
}

Background: Inferior vena cava (IVC) filters are used to prevent massive pulmonary embolism in cases where anticoagulation is contraindicated or has failed. It is usually implanted below the renal veins. In a few cases it is necessary to deploy the filter above them, with theoretical risk of secondary renal failure. Aim: To report the experience with filters located above the renal veins. Patients and Methods: Medical records of all patients with percutaneous suprarenal filters are reviewed. Results: Between May 1993 and May 2007, 361 percutaneous IVC filter procedures were performed. In thirty patients aged 19 to 77 years (average 48 years, 50\% males), they were placed in suprarenal position (8,3\%). Suprarenal IVC filters were implanted in patients with extensive caval thrombosis, renal vein thrombosis extending to cava, displacement of previous IVC filters and double IVC system. Jugular vein approach was the access of choice. Technical success was 100\%, no death or pulmonary embolism occurred. Patients were followed from 1 to 165 months (average 57 months). Eight deaths were recorded, five in patients with cancer. No patient had renal failure on follow up (average creatinine $0.90 \pm 0,26 \mathrm{mg} / \mathrm{dL}$ ). Three patients developed a new deep vein thrombosis (10\%), without pulmonary embolism. Conclusions: In this retrospective analysis of patients, suprarenal placement of IVC filters was not associated to secondary renal failure, and showed good short and long term results (Rev Méd Chile 2008; 136: 1535-41).

(Key w ords: Filters, inferior vena cava; Pulmonary embolism; Vena cava, inferior)

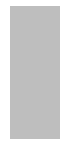

Recibido el 12 de mayo, 2008. Aceptado el 1 de septiembre, 2008.

Sección de Cirugía Vascular y Endovascular, División de Cirugía, Pontificia Universidad Católica de Chile, Instituto Vascular de Santiago.

${ }^{a}$ Enfermera Universitaria

$\mathrm{L}$ a enfermedad tromboembólica venosa es causa importante de morbimortalidad en todo el mundo, siendo su incidencia en población ajustada por edad y sexo de 117 casos por 100.000 habitantes $^{1}$. La terapia habitual de la trombosis

Correspondencia a: Dr. Leopoldo Mariné M. Apoquindo 3990 of 601, Santiago. Fax: (562) 2070718.

E mail: marinepolo@yahoo.com venosa profunda (TVP) y del tromboembolismo pulmonar (TEP) consiste en tratamiento anticoagulante (TAC). Sin embargo, en 10\% a 15\% de los casos el TAC no es posible, fracasa o existen adicionalmente malas condiciones clínicas basales que hacen intolerables un nuevo TEP; en todos ellos está indicado el implante de un filtro de vena cava inferior (FVC) ${ }^{2,3}$. Aunque la ubicación preferente de este dispositivo es bajo la confluencia de las venas renales, existen ocasionalmente razones 
anatómicas que impiden instalarlo en posición infrarrenal ${ }^{4}$.

Existe el temor que la ubicación suprarrenal de los filtros se asocie a mayor riesgo de migración proximal ante mayor diámetro de la vena cava inferior (VCI) en esa localización o la precipitación de insuficiencia renal por extensión de la trombosis hasta el filtro y trombosis secundaria de las venas renales.

El objetivo del presente trabajo es analizar nuestros resultados con FVC de inserción percutánea en ubicación suprarrenal.

\section{MATERIAL Y MÉTODO}

Estudio retrospectivo de pacientes consecutivos en quienes implantamos un FVC sobre el nivel de las venas renales. Se excluyen cuatro casos de FSR tratados entre 1983 y 1993 por denudación yugular, considerándose sólo dispositivos implantados por vía percutánea, a partir de mayo de 1993. Se revisan fichas clínicas, imágenes de control de los procedimientos y protocolos operatorios evaluando características demográficas, indicaciones, detalles técnicos y seguimiento, de acuerdo a estándares de publicación 5 .

Indicación de FSR. Desde 1983, nuestro criterio de colocación de FSR ha sido en todo paciente portador de TVP o TEP con indicación de implante de FVC en quien el implante infrarrenal no sea posible por factores anatómicos: extensión del trombo hasta nivel de las venas renales, TEP recurrente vía venas gonadales o renales, $\mathrm{O}$ ante TEP recurrente por fracaso de filtro infrarrenal previo.

Estudio de imágenes. Durante el período de estudio el eco doppler venoso fue el estudio de imágenes de preferencia en la evaluación de las extremidades inferiores; para la evaluación adecuada de la extensión proximal de la TVP utilizamos una tomografía axial computada complementaria (Figura 1). El TEP fue estudiado mediante tomografía computada helicoidal con fase angiográfica recientemente y mediante cintigrama de ventilaciónperfusión o angiografía en el pasado.

Técnica. La inserción se efectuó en el quirófano bajo condiciones de asepsia y anestesia local, acceso venoso de preferencia vía yugular interna, con técnica de Seldinger y apoyo radioscópico con angiógrafo portátil Siremobil ${ }^{\circledR}$ (Siemens ${ }^{\circledR}$, Alemania) u OEC 9800 ${ }^{\circledR}$ (General Electric ${ }^{\circledR}$, Estados Unidos de Norteamérica). Se mantuvo monitorización de la hemodinamia y oximetría. Previo al despliegue del filtro efectuamos una cavografía para evaluar la anatomía venosa, extensión de trombos en la VCI y medición del diámetro de la vena cava en el sitio planificado para el implante (Figura 2A). Una vez desplegado el FVC (Figura 2B) se retira el sistema introductor y se obtiene hemostasia por compresión directa del sitio de punción.

Éxito técnico. Se define como despliegue completo del dispositivo, en sitio previamente establecido y con adecuado alineamiento respecto a VCI.

Cuidados postoperatorios. Los pacientes son trasladados a su unidad de origen. De no existir contraindicación, la anticoagulación puede reiniciarse o mantenerse sin restricción. Se controló creatinina plasmática postinstalación del FVC.

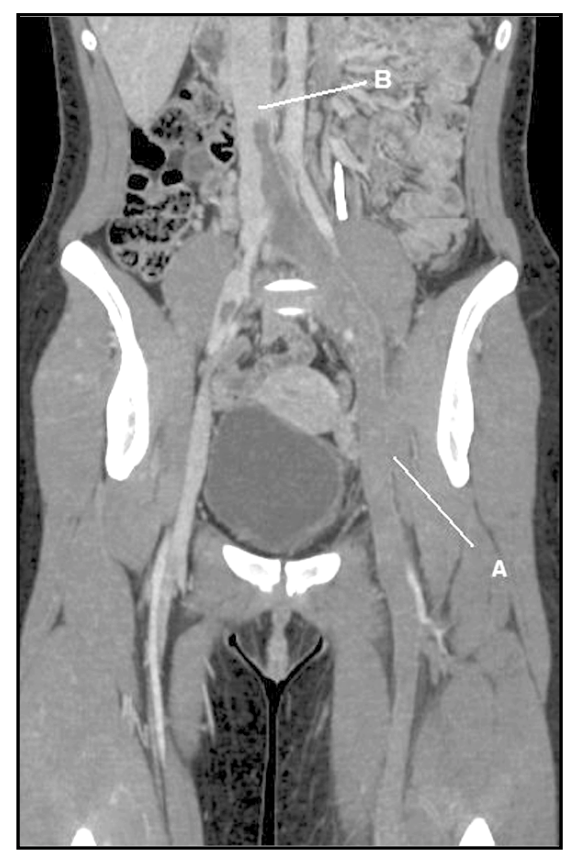

Figura 1. Reconstrucción axial de tomografía axial computada con contraste en fase arterial. (A) Extensa trombosis de venas femorales e ilíacas izquierdas, (B) Muestra extensión de la trombosis a la vena cava inferior en forma pediculada, no adherida a su pared (inestable). 


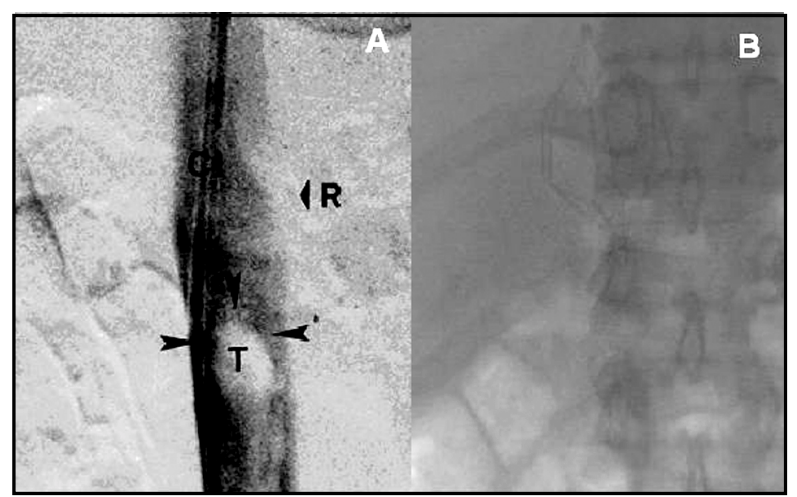

Figura 2. (A) Cavografía muestra trombosis extensa pediculada de la vena cava inferior, C: catéter, R: nivel de confluencia de venas renales, $\mathrm{T}$ y flechas: trombo pediculado, (B) Filtro Trapease ${ }^{\circledR}$ (Cordis, EE.UU.) desplegado sobre el nivel del trombo y de las venas renales.

Seguimiento. Se realizó mediante consulta ambulatoria, contacto telefónico con el paciente o su médico tratante, exámenes de función renal, exámenes selectivos de imágenes según sospecha clínica y eventualmente obtención de certificados de defunción emitidos por el Servicio de Registro Civil e Identificación.

Estadística. Se entregan cifras descriptivas.

\section{RESULTADOS}

Entre mayo de 1993 y mayo de 2007 implantamos un total de 361 FVC en 361 pacientes, de los cuales 30 corresponden a filtros suprarrenales (FSR) (8,3\%). El promedio de edad de este subgrupo fue de $47,7 \pm 16,8$ años (rango 19-77) y sexo masculino en 15 casos (50\%). Las patologías asociadas se describen en la Tabla 1. Las más

Tabla 1. Patología asociada en pacientes tratados con FSR

\begin{tabular}{|lr|}
\hline Patología asociada & Número de pacientes (\%) \\
\hline Neoplasias & $14(46,7)$ \\
Enfermedades cardiovasculares & $8(26,7)$ \\
Enfermedades del sistema digestivo & $7(23,3)$ \\
Enfermedades del sistema genitourinario & $7(23,3)$ \\
Enfermedades psiquiátricas & $7(23,3)$ \\
Enfermedades hematológicas & $5(16,7)$ \\
Insuficiencia renal & $5(16,7)$ \\
Hemodiálisis & $1(3,3)$ \\
Enfermedades endocrinas, nutricionales, metabólicas & $3(10,0)$ \\
Enfermedades ginecológicas & $3(10,0)$ \\
Enfermedades del sistema respiratorio & $2(6,7)$ \\
Enfermedades del sistema ME y tejido conjuntivo & $2(6,7)$ \\
Enfermedades del sistema nervioso & $1(3,3)$ \\
Aneurisma aorta abdominal complicado & $1 \quad(3,3)$ \\
\hline
\end{tabular}

ME: músculo-esquelético 
frecuentes son las enfermedades neoplásicas que corresponde a casi la mitad de los casos de la serie (14 casos, 46,7\%). Una paciente portadora de enfermedad de Wegener se encontraba en hemodiálisis al momento de la indicación de FVC.

Las formas de presentación clínica y las indicaciones de implante se describen en extenso en la Tabla 2. Todos los pacientes presentan alguna manifestación tromboembólica. Las indicaciones de interrupción de VCI más frecuentes fueron la presencia de trombosis ascendente de VCI y un TEP reciente, y la trombosis en VCI inestable (pediculada, flotante) con inminencia de TEP. Dieciocho casos (60\%) presentaban TEP al momento de la indicación de FVC.

Respecto de las indicaciones de colocación del filtro sobre las venas renales (Tabla 3), la indicación más frecuente fue trombosis extensa de la VCI hasta la confluencia de las venas renales, la que, por falta de espacio, impide su despliegue infrarrenal (24 casos). En cuatro pacientes la indicación fue la presencia de trombosis de vena renal con extensión pediculada a VCI. Finalmente dos pacientes fueron diagnosticados con TEP sin identificarse TVP concomitante en estudios de imágenes a su ingreso y en los cuales se realizó implante profiláctico de FSR: uno de ellos presentaba malposición por angulación de FVC infrarrenal insertado previamente en otra institución y TEP recurrente, el segundo era portador de un doble sistema de VCI asociado a TEP crónico grave, en preparación para una tromboendarterectomía de arteria pulmonar.

En 25 casos utilizamos el acceso yugular $(85,1 \%)$, en un caso la vena subclavia y en 4 casos la vena femoral (13,3\%). En estos últimos 4 pacientes la extensión de la TVP ilíaca a la VCI perirrenal fue hallazgo de la cavografía diagnóstica realizada durante la intervención, correspondiendo éstos a casos realizados en los primeros años de la serie, previo al uso liberal de la tomografía. Los diferentes tipos de filtros utilizados se detallan en la Tabla 4. Sólo empleamos FVC permanentes. La selección del tipo de filtro dependió de la preferencia del tratante o la disponibilidad al momento del implante y del diámetro de la VCI.

El éxito técnico fue de 100\%. No hubo morbilidad sistémica asociada ni en el sitio de punción, y no observamos un incremento significativo de la

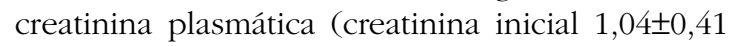
$\mathrm{mg} / \mathrm{dL}$, creatinina posterior $1,08 \pm 0,60 \mathrm{mg} / \mathrm{dL}, \mathrm{p}$

\section{Tabla 2. Formas de presentación e indicaciones de colocación de filtro de vena cava inferior}

\section{Indicación}

Trombo en VCI y TEP reciente

Trombosis de VCI pediculado inestable

Contraindicación de TAC

TEP y mala reserva pulmonar

Fracaso de tratamiento con TEP recurrente

Necesidad de cirugía pronta

Número de pacientes (\%)

$9(30,0)$

$7(23,3)$

$7 \quad(23,3)$

$3(10,0)$

$2(6,7)$

$2(6,7)$

\section{Tabla 3. Indicaciones de colocación de FVC en posición suprarrenal}

\begin{tabular}{|lc|}
\hline Indicación FSR & Número de pacientes (\%) \\
\hline Trombosis extensa de VCI cercana a venas renales & $24(80,0)$ \\
Trombosis venal renal con extensión pediculada a VCI & $4(13,3)$ \\
Malposición de filtro previo y TEP actual & $1(3,3)$ \\
Doble sistema de VCI y TEP crónico & $1 \quad(3,3)$ \\
\hline
\end{tabular}


Tabla 4. Tipos de filtros suprarrenales utilizados

\begin{tabular}{|llrl|}
\hline Tipo de filtro & Compañía & n & (\%) \\
\hline Trapease/Optease $^{\circledR}$ & Cordis (EE.UU.) & 11 & $(36,6 \%)$ \\
Venatech $^{\circledR}$ & Braun (EE.UU.) & 10 & $(33,3 \%)$ \\
Greenfield Titanio $^{\circledR}$ & Boston Sci (EE.UU.) & 8 & $(26,7 \%)$ \\
Gunther-Tulip $^{\circledR}$ & Cook (EE.UU.) & 1 & $(3,3 \%)$ \\
\hline
\end{tabular}

Tabla 5. C ausas de muerte en pacientes sometidos a implante de FSR

\begin{tabular}{|c|c|}
\hline Periodo & Causa $(n, \%)$ \\
\hline Intervención & 0 \\
\hline 30 días & 0 \\
\hline Seguimiento & $8 \quad(26,7 \%)$ \\
\hline Cáncer terminal & 5 (leucemia, linfoma, cáncer vesical, gástrico y cérvico-uterino) \\
\hline Infección respiratoria y sepsis & 1 (operado de AAA roto) \\
\hline Infección respiratoria y EBOC & 1 \\
\hline Isquemia miocárdica & 1 \\
\hline
\end{tabular}

AAA: aneurisma de la aorta abdominal, EBOC: enfermedad bronquial obstructiva crónica.

$=0,82$ ) o mortalidad asociada al procedimiento, hospitalaria o a 30 días (Tabla 5).

Se obtuvo seguimiento en todos los casos. Éste fue 57,3 meses en promedio (rango 1 y 165 meses). Dos pacientes fallecieron tempranamente en este período: un paciente portador de cáncer vesical fallece a los 33 días del implante por sepsis urinaria, y

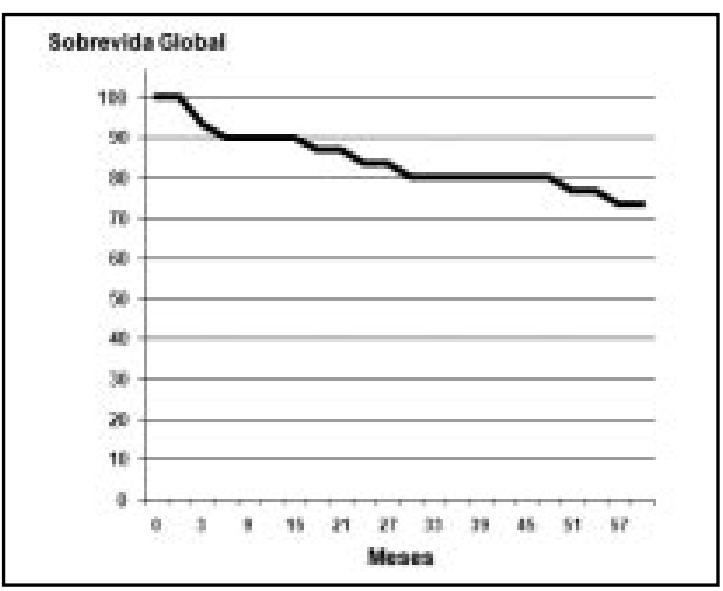

Figura 3. Tabla de sobrevida de filtros suprarrenales. otro paciente portador de enfisema pulmonar e insuficiencia renal fallece a los 78 días por infección respiratoria. Cinco de los 8 pacientes fallecidos en el seguimiento alejado eran portadores de cáncer (Tabla 5). Durante el seguimiento alejado 3 pacientes presentaron TVP de extremidades inferiores (10\%), uno de ellos portador de cáncer gástrico avanzado. Doce de los pacientes sobrevivientes $(54,5 \%)$ se mantienen bajo TAC, sin complicaciones asociadas a éste. En 6 casos se demostró algún tipo de trombofilia. No hemos encontrado otras complicaciones alejadas reportadas en la literatura, como migración del FVC o fistulización a órganos vecinos. Tampoco se detectó insuficiencia renal, encontrándose la creatinina plasmática normal en los 22 pacientes sobrevivientes al cierre del estudio (creatinina $0,90 \pm 0,26 \mathrm{mg} / \mathrm{dL}$ ). No se registraron TEP recurrentes. La curva de sobrevida se grafica en la Figura 3.

\section{DisCUSIÓN}

El FVC ha demostrado ser una alternativa eficaz en pacientes en los cuales el tratamiento anticoagulante 
esté contraindicado o sea insatisfactorio. Su despliegue se efectúa normalmente en posición infrarrenal, siendo ésta impracticable en una proporción baja de pacientes $^{4}$, ya sea por trombosis extensa de la cava hasta la vecindad de las venas renales ${ }^{6}$, trombosis pediculada hacia la cava desde las venas gonadales $^{7,8}$ o renales 9 o, por otro lado, cuando la ubicación suprarrenal es mejor alternativa de protección por duplicidad de $\mathrm{VCI}^{10} \mathrm{o}$ ante problemas en filtros infrarrenales previos con TEP recurrentes ${ }^{11,12}$.

La función protectora de los FVC se logra mediante la interceptación de material embólico proveniente de las venas de las extremidades o pelvis, o al establecer límite a la progresión ascendente de trombosis ya establecida en la cava. De esta manera, la trombosis hasta el nivel del filtro en ubicación suprarrenal determinaría, teóricamente, un riesgo de trombosis secundaria de las venas renales y la consecuente insuficiencia renal. Sin embargo, esto no ha sido observado, ya sea en modelos animales de ligadura de vena cava suprarrenal ${ }^{13}$, como tampoco en estudios clínicos retrospectivos de comparación de morbimortalidad de filtros sobre y bajo las venas renales, demostrándose similares resultados en ambas localizaciones ${ }^{14,15}$. La explicación más probable para la ausencia de desarrollo de insuficiencia renal por la interferencia de flujo de la VCI que pueda ocasionar el FVC, es el establecimiento de un drenaje venoso alternativo a través de inversión del flujo de venas colaterales afluentes de las venas renales, como serían las venas suprarrenales o gonadales, sobre todo al lado izquierdo. Excepcionalmente se han descrito casos de insuficiencia renal en el subgrupo de pacientes con cáncer avanzado y filtro suprarrenal, cuyo mecanismo más probable sería el desarrollo de trombosis de esta red venosa colateral en el contexto del estado de trombofilia propio de una neoplasia avanzada ${ }^{16}$. En nuestra serie no observamos variación de creatinina plasmática posterior al procedimiento, manteniéndose en rango normal durante el seguimiento alejado de todos los pacientes sobrevivientes.
En cuanto al diámetro de la VCI, éste es mayor por sobre la confluencia de las venas renales. Normalmente el diámetro promedio de la VCI infrarrenal es de 20 a $22 \mathrm{~mm}$, a nivel suprarrenal entre 24 y 26, y supradiafragmática entre 30 y $32 \mathrm{~mm}$. En $2 \%$ a 3\% de los pacientes es posible observar diámetros aún mayores (megacavas), las que se asocian a insuficiencia cardiaca congestiva, cor pulmonale e insuficiencia tricuspídea, ninguna de estas situaciones se presentó en esta serie. La selección del dispositivo adecuado para el diámetro particular de cada VCI evita su migración o embolización ${ }^{17}$, en esta serie el diámetro fue menor de $28 \mathrm{~mm}$ por lo que fue innecesario utilizar dispositivos especiales para VCI grandes tipo Bird's Nest ${ }^{\circledR}$ (Cook, EEUU).

En el seguimiento de nuestra serie identificamos 3 casos de TVP recurrente (10\%). Esta es una incidencia ligeramente mayor que la reportada anteriormente por nuestro grupo en los FVC en todas localizaciones, tanto percutáneos ${ }^{18,19}$ como por denudación venosa ${ }^{20}$, explicable probablemente por el mayor porcentaje de pacientes con neoplasia de esta serie, ocurriendo ésta pese a que más de la mitad de los pacientes se encuentran en tratamiento anticoagulante.

Consideramos que la ausencia de morbimortalidad asociada al dispositivo observada en esta experiencia es consecuencia de una técnica de instalación prolija, que debe considerar:

1. Acceso yugular de elección. 2. Navegación cuidadosa de la guía para evitar contactar el trombo en la vena cava. 3. Cavografía rutinaria con técnica de sustracción digital de manera de: evaluar extensión del trombo, anomalías de VCI, ubicación de las venas tributarias (renales y hepáticas) y establecer así el sitio más adecuado para desplegar el filtro. 4. En ausencia de escáner abdominal previo a la intervención, realizar medición del diámetro de la VCI en la cavografía para la selección del FVC más adecuado.

Basados en nuestras observaciones, el implante de un FSR es un procedimiento seguro, con buenos resultados a corto y largo plazo, y que debe ser indicado en situaciones clínicas especificas para profilaxis segura del TEP. 


\section{REFERENCIAS}

1. Silverstein MD, Heit JA, Mohr DN, Petterson TM, O'Fallon WM, MeLton LJ $3^{\mathrm{RD}}$. Trends in the incidence of deep vein thrombosis and pulmonary embolism: a 25-year population-based study. Arch Intern Med 1998; 158: 585-93.

2. British Committee for Standards in Haematology Writing Group, Baglin TP, Brush J, StreifF M. Guidelines on use of vena cava filters. Br J Haematol 2006; 134 : 590-5.

3. Streiff MB. Vena Cava Filters: a Comprehensive Review. Blood 2000; 95: 3669-77.

4. Grassi CJ, Swan Tl, Cardella Jf, Meranze SG, Oglevie SB, OMARY RA ET AL. Quality Improvement Guidelines for Percutaneous Permanent Inferior Vena Cava Filter Placement for the Prevention of Pulmonary Embolism. J Vasc Inter Radiol 2001; 12: 137-41.

5. The Participants in the Vena Cava Filter Consensus Conference. Recommended Reporting Standards for Vena Cava Filter Placement and Patient Follow-Up. J Vasc Interv Radiol 2003; 14: S427-32.

6. Radomski JS, Jarrell BE, Carabasi RA, Yang SL, Koolpe H. Risk of Pulmonary Embolus with Inferior Vena Cava Thrombosis. Am Surg 1987; 53: 97-101.

7. Clarke CS, Harlin SA. Puerperal Ovarian vein Thrombosis with Extension into the Inferior Vena Cava. Am Surg 1999; 65: 147-50.

8. Abujudeh H, Lim H. Emergency Suprarenal Inferior Vena Cava Filter Placement in Ovarian Vein Thrombosis. Emergency Radiology 2004; 10: 270-2.

9. O'Brien AA, O’Donnell JP, Keogh JA. Renal Vein Thrombosis with Recurrent Pulmonary Emboli in the Nephrotic Syndrome: Use of the Greenfield Filter. Postgrad Med J 1986; 62: 223-5.

10. Mano A, Tatsumi T, Sakai $\mathrm{H}$, Imoto $\mathrm{Y}$, Nomura $\mathrm{T}$, NishiKawa S ET AL. A Case of Deep Venous Thrombosis With a Double Inferior Vena Cava Effectively Treated by Suprarenal Filter Implantation. Jpn Heart J 2004; 45: 1063-9.
11. Kazmers A, Ramnauth S, Williams M. Intraoperative Insertion of Greenfield Filters: Lessons Learned in a Personal Series of 152 Cases. Am Surg 2002; 68: 87782.

12. Haase SC, Wolk SW, Lampman RM, Sarosi MG, Misare BD, ErLandson EE et al. Patient Outcomes Following Placement of a Second Inferior Vena Cava Filter: Case Reports. Vasc Endovascular Surg 1988; 32: 6337.

13. Peyton JW, Stewart JR, Greenfield LJ, Crute SL. Hemodynamics and renal function following experimental suprarenal vena caval occlusion. Surg Gynecol Obstet 1982; 155: 37-42.

14. Greenfield LJ, Proctor MC. Suprarenal Filter Placement. J Vasc Surg 1998; 28: 432-8.

15. Athanasoulis CA, Kaufman JA, Halpern EF, Waltman AC, Geller SC, Fan CM. Inferior Vena Cava Filters: Review of a 26-year Single-Center Clinical Experience. Radiology 2000; 216: 54-66.

16. Marcy Py, Magne N, Frenay M, Bruneton JN. Renal Failure Secondary to Thrombotic Complications of Suprarenal Inferior Vena Cava Filter in Cancer Patients. Cardiovasc Intervent Radiol 2001; 24: 2579.

17. Prince MR, Noveldine RA, Athanasoulis CA, Simon M. The Diameter of the Inferior Vena Cava and Its Implications for the Use of Vena Caval Filters. Radiology 1983; 149: 687-9.

18. Mertens R, Valdes F, Kramer A, Vergara J. Interrupción de vena cava inferior mediante filtros: Indicaciones y resultados en 111 pacientes. Rev Méd Chile 1998; 126: 655-60.

19. Arriagada I, Mertens R, Valdés F, Kramer A, Marine L, Bergoeing M ET AL. Interrupción de Vena Cava Inferior Mediante Filtros de Inserción Percutánea: Indicaciones y Resultados en 287 Pacientes. Rev Méd Chile 2007; 135: 351-8.

20. Valdés F, Kramer A, Turner E. Interrupción quirúrgica de la vena cava Inferior. Resultado en 36 Casos. Rev Chil Cardiol 1986; 23: 40. 